\title{
ON EMBEDDING OF GROUP RINGS OF RESIDUALLY TORSION FREE NILPOTENT GROUPS INTO SKEW FIELDS
}

\author{
A. EIZENBUD AND A. I. LICHTMAN
}

\begin{abstract}
ABSTKACT. It is proven that the group ring of an amalgamated free product of residually torsion free nilpotent groups is a domain and can be embedded in a skew field. This is a generalization of J. Lewin's theorem, proven for the case of free groups. Our proof is based on the study of the Malcev-Neumann power series ring $K\langle G\rangle$ of a residually torsion free nilpotent group $G$. It is shown that its subfield $D$, generated by the group ring $K G$, does not depend on the order of $G$ for many kinds of orders and the study of $D$ can be reduced in some sense to the case when $G$ is nilpotent.
\end{abstract}

1. Introduction. Let $G$ be an ordered group and let $K G$ be its group ring over a commutative field $K$. (The term "field" throughout this paper is used in the sense of "skew field.") It is known that $K G$ can be embedded into the Malcev-Neumann power series ring $K\langle G\rangle$ which is in fact a field. In this paper we study some properties of this field $K\langle G\rangle$ in the case when $G$ is a residually torsion free nilpotent group, or more generally, when $G$ contains a descending series of normal subgroups

$$
G=N_{1} \supseteq N_{2} \supseteq \cdots
$$

such that

$$
\bigcap_{i=1}^{\infty} N_{i}=1
$$

and all the groups $G_{i}=G / N_{i}(i=1,2, \ldots)$ are ordered in such a way that the natural homomorphisms $G / N_{i+1} \rightarrow G / N_{i}$ are ordered group homomorphisms. It is easy to show (Lemma 4.1) that the group $G$ can be ordered in such a way that all the homomorphisms $G \rightarrow G / N_{i}=G_{i}$ are homomorphisms of ordered groups.

Our main results are related to the case when all the quotient groups $G_{i}=G / N_{i}$ in (1.1) are torsion free nilpotent. We prove that in this case the field $D$ does not depend on the choice of series (1.1) in $G$. More precisely, let $H_{j}(j=1,2, \ldots)$ be the second series in $G$ with torsion free nilpotent quotient groups $G / H_{j}$ and unit intersection and let $G$ be ordered in such a way that all the natural homomorphisms $G \rightarrow G / H_{j}$ are

Received by the editors March 31, 1985 and, in revised form, November 26, 1985.

1980 Mathematics Subject Classification. Primary 20C07, 16A27; Secondary 16A08, 16A39.

Key words and phrases. Group rings, skew fields, free products, ordered groups, residually nilpotent groups.

The second author was partially supported by NSF Grant No. D14S- 8400445 . 
homomorphisms of ordered groups. Let $D^{\prime}$ be the subfield generated by $K G$ in the new Malcev-Neumann power series ring. Then the fields $D$ and $D^{\prime}$ are isomorphic (Theorem 6.2).

This result was motivated by J. Lewin's theorem in [1] which states that in the case when $G$ is a free group, the field $D$ is the universal field of fractions for $K G$. We do not know whether this remains true in the case when $G$ is an arbitrary residually torsion free nilpotent group, and it is unknown too whether a group ring of such a group has a universal field of fractions, but the conclusion of Theorem 6.2 may support this conjecture.

Lewin also proved that for every subgroup $F$ of a free group $G$ the subfield generated by $K F$ is isomorphic to the universal field of fractions $K F$. Using these results and the theorems of Cohn that a coproduct of fields over a subfield is a fir and that a fir has a universal field of fractions (see $[2,3]$ ), Lewin proves that if $G_{1}$ and $G_{2}$ are free groups, then the group ring of the amalgamated free product $G_{1} *{ }_{F} G_{2}$ is a domain and can be embedded in a field. We apply Theorem 6.2 together with Lewin's idea of independency preserving embedding $[\mathbf{1}, \mathbf{4}]$ in order to prove the following result.

THEOREM 6.3. Let $G_{1}$ and $G_{2}$ be residually torsion free nilpotent groups, with $G=G_{1}{ }_{F} G_{2}$ their free product with an amalgamated subgroup $F$. Then the group ring $K G$ contains no zero divisors and can be embedded in a field.

The appropriate result for HNN-extensions follows easily.

We describe now some of our other results on prime matrix ideals and specializations related to $K\langle G\rangle$.

Let $D$ and $D_{i}(i=1,2, \ldots)$ denote the subfields of $K\langle G\rangle$ and $K\left\langle G_{i}\right\rangle$ generated by $K G$ and $K G_{i}$ respectively. It follows from Cohn's Theorem 7.5.3 in [5] (see also Malcolmson [6]) that the fields $D$ and $D_{i}$ are defined by prime matrix ideals $\mathscr{P}$ and $\mathscr{P}_{i}$ over $K G(i=1,2, \ldots)$. We prove in Theorem 4.1 that

$$
\mathscr{P}_{1} \supseteq \mathscr{P}_{2} \supseteq \cdots
$$

and

$$
\mathscr{P}=\bigcap_{i=1}^{\infty} \mathscr{P}_{i} .
$$

The proof of this theorem is based first of all on Proposition 4.1 which allows us to construct specializations $K\langle G\rangle \rightarrow K\left\langle G_{i}\right\rangle$ and on Proposition 2.1 which is related to the case of an arbitrary ring $R$ and states that relation (1.4) is equivalent to the following two conditions:

(i) for every given $i$ there exists a specialization $\Theta_{i}: D \rightarrow D_{i}$,

(ii) for any given elements $x_{1}, x_{2}, \ldots, x_{k}$ an index $i$ can be found such that $x_{1}, x_{2}, \ldots, x_{k}$ belong to the domain $T_{i}$ of the specialization $\Theta_{i}$.

When both conditions (1.3) and (1.4) hold, we prove Proposition 2.2 which states that $D$ is a union of an ascending series of the subrings $T_{i}$. The results of this type can be used to reduce the study of the field $D$ to the study fo fields $D_{i}$ (see, for instance, $[7-11])$. 
Finally, we consider another embedding for $K G$ taking an ultraproduct $\left(\prod_{i=1}^{\infty} D_{i}\right) / \mathscr{F}$. Then $K G$ is embedded into the field $\left(\prod_{i=1}^{\infty} D_{i}\right) / \mathscr{F}$ and generates in it a subfield $\Delta$. We prove in Theorem 6.1 that $\Delta$ is isomorphic to the field $D$, obtained from the Malcev-Neumann embedding.

\section{2.}

2.1. In this section we give a brief account of some results about prime matrix ideals and specializations; the detailed exposition can be found in Cohn's book [5, Chapter 7].

Let $R$ be a ring. If there exists a homomorphism $\psi$ of $R$ into a field $S$ such that $S$ is generated by the ring $\psi(R)$, then $S$ is called an $R$-field. If $\psi$ is injective, then $S$ is called a field of fractions of $R$. A specialization $\Theta$ between two $R$-fields $S$ and $L$ is a (surjective) $R$-ring homomorphism of a local subring $S_{0} \subseteq S$ on $L$. The $R$-field and their specializations form a category which is equivalent to the category of prime matrix ideals over $R$ with embeddings as the maps in the category. We refer the reader to [5] for the definition of the prime matrix ideal; here we need only the following theorem of Cohn:

Let $S$ be an $R$-field. Then the set of matrices whose images under the homomorphism $R \rightarrow S$ become singular over $S$ form a prime matrix ideal and conversely if a prime matrix ideal $\mathscr{P}$ over $R$ is given, then there exists an $R$-field $S$ such that $\mathscr{P}$ coincides with all the matrices which are mapped into the singular ones under the homomorphism $R \rightarrow S$ (see [5, Theorem 7.5.3 and 6]).

If a prime matrix ideal $\mathscr{P}$ is given then the field $S$ is constructed in the following way. First one has to construct the universal inverting ring $R_{\mathscr{P}}$ for the complement of $\mathscr{P}$ (see [5]). The ring $R_{\mathscr{P}}$ is local; let $J\left(R_{\mathscr{P}}\right)$ be its radical. Then $S$ is isomorphic to the quotient ring $R_{\mathscr{P}} / J\left(R_{\mathscr{P}}\right)$.

The last result together with Theorem 7.2.3 in [5] implies immediately: Let $S_{i}$ $(i=1,2)$ be two $R$-fields and $\mathscr{P}_{i}(i=1,2)$ the related prime matrix ideals. Then there exists a specialization $\Theta: S_{1} \rightarrow S_{2}$ of $R$-fields iff $\mathscr{P}_{2} \supseteq \mathscr{P}_{1}$.

2.2. Proposition 2.1. Let $D$ be an $R$-field and $\mathscr{P}$ the corresponding prime matrix ideal. Consider a family of $R$-fields $D_{i}$ which are defined by matrix ideals $\mathscr{P}_{i}(i \in I)$. Then the relation

$$
\mathscr{P}=\bigcap_{i \in I} \mathscr{P}_{i}
$$

holds if and only if the following two conditions are satisfied:

(i) For every given $i$ there exists a specialization $\Theta_{i}: D \rightarrow D_{i}$.

(ii) For every given elements $x_{1}, x_{2}, \ldots, x_{k} \in D$ an index $i$ can be found such that $x_{1}, x_{2}, \ldots, x_{k}$ belong to the domain $T_{i}$ of the specialization $\Theta_{i}$.

PROOF. Assume that (2.1) holds. Then (i) follows from the condition $\mathscr{P} \subseteq \bigcap_{i \in I} \mathscr{P}_{i}$. We now prove that (ii) also holds. Thus let $x_{1}, x_{2}, \ldots, x_{k}$ be given nonzero elements of $D$. Pick the elements $t_{1}, t_{2}, \ldots, t_{k}$ in $R_{\mathscr{P}}$ such that the image of $t_{\alpha}$ in $R_{\mathscr{P}} / J\left(R_{\mathscr{P}}\right)$ $\simeq D$ is $x_{\alpha}$. 
We can find a finite number of matrices $A_{1}, A_{2}, \ldots, A_{n} \notin \mathscr{P}$ such that the elements $t_{\alpha}(\alpha=1,2, \ldots, k)$ occur as entries of these matrices and their inverses. If $A=A_{1} \oplus A_{2} \oplus \cdots \oplus A_{n}$ is the diagonal sum of these matrices, then $A \notin \mathscr{P}$ since $\mathscr{P}$ is prime and in fact the elements $t_{\alpha}(\alpha=1,2, \ldots, k)$ occur as the entries of $A$ and $A^{-1}$. We now obtain from (2.1) that there exists an $i \in I$ such that $A \notin \mathscr{P}_{i}$. This implies easily that the elements $t_{1}, t_{2}, \ldots, t_{k}$ belong to some subring $U_{i}$ of $R_{\mathscr{P}}$ which is a homomorphic image of $R_{\mathscr{P}_{i}}$; clearly, $U_{i}$ must be local and $U_{i} / J\left(U_{i}\right) \simeq$ $R_{\mathscr{P}_{i}} / J\left(R_{\mathscr{P}_{i}}\right) \simeq D_{i}$.

Finally, the homomorphism $U_{i} \rightarrow U_{i} / J\left(U_{i}\right) \simeq D_{i}$ can be factored through the homomorphism $U_{i} \rightarrow U_{i} / U_{i} \cap J\left(R_{\mathscr{P}}\right)$; the last ring is a local subring of $R_{\mathscr{P}} / J\left(R_{\mathscr{P}}\right)$ $\simeq D$ which contains the elements $x_{1}, x_{2}, \ldots, x_{k}$. We denote it by $T_{i}$ and see that $T_{i} / J\left(T_{i}\right) \simeq U_{i} / J\left(U_{i}\right) \simeq D_{i}$; this completes the proof of (ii).

Conversely, let (i) and (ii) hold. Then (i) implies that $\mathscr{P} \subseteq \mathscr{P}_{i}(i \in I)$. Now, if the inclusion $\mathscr{P} \subseteq \bigcap_{i \in I} \mathscr{P}_{i}$ is proper, then there exists a matrix $B$ which is mapped into an invertible matrix under the homomorphism $\psi: R \rightarrow D$ but $B \in \bigcap_{i \in I} \mathscr{P}_{i}$, i.e., $B$ is mapped into singular matrices under the homomorphisms $\psi_{i}: R \rightarrow D_{i}$.

Let $C$ be a matrix such that $\psi(B) C=1$ and let $r_{1}, r_{2}, \ldots, r_{s}$ be all the entries of $\psi(B), C$. Find a specialization $\Theta_{i}$ of $R$-fields $D$ and $D_{i}$ such that $r_{1}, r_{2}, \ldots, r_{s}$ belong to the domain $T_{i}$ of $\Theta_{i}$. Then

$$
1=\Theta_{i}(\psi(B) C)=\psi_{i}(B) \psi_{i}(C),
$$

which contradicts the singularity of $\psi_{i}(B)$ in $D_{i}$.

We are interested now in the special case of the situation which was considered in Proposition 2.1.

Proposition 2.2. Let

$$
\mathscr{P}_{1} \supseteq \mathscr{P}_{2} \supseteq \cdots
$$

be a descending chain of prime matrix ideals over $R$ and $\mathscr{P}=\bigcap_{i=1}^{\infty} \mathscr{P}_{i}$. Let $D_{i}$ be the $R$-field, defined by the prime matrix ideal $\mathscr{P}_{i}(i=1,2, \ldots, n)$. Then an $R$-field $D$ is defined by the prime matrix ideal $\mathscr{P}$ iff it contains an ascending series of local $R$-subrings

$$
T_{1} \subseteq T_{2} \subseteq \cdots
$$

such that

$$
\bigcup_{i=1}^{\infty} T_{i}=D
$$

and

$$
T_{i} / J\left(T_{i}\right) \simeq D_{i} \quad(i=1,2, \ldots) .
$$

Remark. It is worth remarking that Proposition 2.2 states that $D$ is defined by the prime matrix ideal $\mathscr{P}$ iff it is a union of an ascending series of domains of specializations $\Theta_{i}: D \rightarrow D_{i}(i=1,2, \ldots)$.

Proof of Proposition 2.2. Assume that the conditions of the assertion are satisfied. Then conditions (i) and (ii) of Proposition 2.1 follow and we conclude from Proposition 2.1 that the prime matrix ideal, defined by $D$, coincides with $\mathscr{P}$. 
Conversely, let $D$ be defined by the prime matrix ideal $\mathscr{P}=\bigcap_{i=1}^{\infty} \mathscr{P}_{i}$. Then we have for the complements $C(\mathscr{P})$ and $C\left(\mathscr{P}_{i}\right)$ of the prime matrix ideals $\mathscr{P}$ and $\mathscr{P}_{i}$ $(i=1,2, \ldots)$ respectively:

$$
C\left(\mathscr{P}_{1}\right) \subseteq C\left(\mathscr{P}_{2}\right) \subseteq \cdots ; \quad C(\mathscr{P})=\bigcup_{i=1}^{\infty} C\left(\mathscr{P}_{i}\right) .
$$

Let $U_{i}$ be the subring of the universal inverting ring $R_{\mathscr{P}}$ generated by the entries of all the matrices from $C\left(\mathscr{P}_{i}\right)$ and of their inverses. Clearly,

$$
U_{1} \subseteq U_{2} \subseteq \cdots ; \quad R_{\mathscr{P}}=\bigcup_{i=1}^{\infty} U_{i} \cdots
$$

As in the proof of Proposition 2.1, we obtain that every subring $U_{i} \subseteq R_{\mathscr{P}}$ is local and $U_{i} / J\left(U_{i}\right) \simeq D_{i}$; once again, $T_{i}=U_{i} \mid U_{i} \cap J\left(R_{\mathscr{P}}\right)$ is a local subring of $R_{\mathscr{P}} / J\left(R_{\mathscr{P}}\right)$ $\simeq D$ which is the domain of the specialization $D \rightarrow D_{i}(i=1,2, \ldots)$ and $(2.5)$ follows. Finally, (2.3) and (2.4) follow from (2.7) and the definition of $T_{i}$.

3.

3.1. Let $G$ be an arbitrary ordered group; denote by $K\langle G\rangle$ the Malcev-Neumann power series ring of $G$ over $K$. We remind the reader that an arbitrary element of $K\langle G\rangle$ has a form $u=\sum \alpha_{x} x$, where its support Supp $u=\left\{x \in G \mid \alpha_{x} \neq 0\right\}$ is a well-ordered subset of $G$; the addition and multiplication are defined in a natural way. We refer the reader to [12] for all the proofs and definitions; here we only point out that the proof of the correctness of the operations in $K\langle G\rangle$ is based on the following facts:

Let $V_{1}$ and $V_{2}$ be two well-ordered subsets of $G$. Then

(i) the subset $V_{1} V_{2}$ is well ordered too,

(ii) for given elements $v_{i} \in V_{i}(i=1,2)$ there exists only a finite number of elements in $V_{1} V_{2}$ which are equal to the element $v_{1} v_{2}$.

The ring $K\langle G\rangle$ is in fact a field (see [12]). The same argument gives the following assertion.

LEMMA 3.1. Let $V$ be the subset of elements of $K\langle G\rangle$ which have 1 as the minimal element in their support. Then $V$ is a subgroup of the multiplicative group of $K\langle G\rangle$.

Proof. Let $v \in V$. Then $v=\alpha_{1}(1-u)$, where $\alpha_{1} \neq 0$ and all the elements in Supp $u$ are greater than 1 . But it follows easily from statements (i) and (ii) that in the infinite series $1+u+u^{2}+\cdots$ a given element $g \in G$ can occur only in the support of a finite number of terms and this series thus defines an element of $K\langle G\rangle$; furthermore, this implies that

$$
v^{-1}=\alpha_{1}^{-1}\left(1+u+u^{2}+\cdots\right) .
$$

It also follows from statements (i) and (ii) that $U$ is multiplicatively closed.

Now let $G \stackrel{\psi}{\rightarrow} H$ be a surjective homomorphism of ordered groups $G$ and $H$, and let $X$ be a transversal of $H$ in $G$. Let $N=\operatorname{ker} \psi$. The homomorphism $\psi$ induces the homomorphism $K G \rightarrow K H$ with kernel $\omega(K N) K$, where $\omega(K N)$ is the augmentation ideal of $K N$; it is generated by all the elements $h-1(h \in N)$. Consider an 
element $v \in K\langle G\rangle$ such that its support has a finite or a void intersection with every coset $x N(x \in X)$. It follows immediately that such an element can be represented in the form

$$
v=\sum_{i \in I} \lambda_{i} x_{i}
$$

where $\lambda_{i}(i \in I)$ are elements of the group ring $K N$ and it is important that the set $\left\{x_{i} \mid i \in I\right\}$ is a well-ordered subset of $X$. Conversely, every element which has a representation of the form (3.1) has the property that $\operatorname{Supp} v \cap x N$ is finite or void for every $x \in X$. It is easy to see that the existence of representation (3.1) for a given element does not depend on the choice of the transversal.

PROPOSITION 3.1. Let $S$ be the set of elements which have representation of the form (3.1). Then $S$ is a local ring whose radical is $\omega(K N) S$ and $S / \omega(K N) S$ is isomorphic to $K\langle H\rangle$.

Proof. It is immediate that $\omega(K N) S$ coincides with the subset of elements of $S$ whose coefficients $\lambda_{j}(j \in J)$ in representation (3.1) belong to $\omega(K N)$. We easily conclude from this that the quotient ring $S / \omega(K N) S$ is isomorphic to the field $K\langle H\rangle$ and it remains to prove that if $v \notin \omega(K N) S$ then $v$ is invertible. If $v$ is such an element and $x_{i_{1}}$ is the minimal element among the elements $x_{i}(i \in I)$ in (3.1) then if necessary we can replace the element $v$ by the element $v x_{i_{1}}^{-1}$ and assume that the set $x_{i}(i \in I)$ has 1 as its minimal element (and is well ordered). Furthermore, we can find an element $\sum_{i \in I} \alpha_{i} x_{i}\left(i \in I, \alpha_{i} \in K\right)$ such that

$$
v \equiv \sum_{i \in I} \alpha_{i} x_{i} \quad(\bmod \omega(K N) S) .
$$

If $\bar{x}$ denotes the image of the element $x$ under the natural homomorphism $S \rightarrow S / \omega(K N) S$, then the element

$$
\bar{o} \neq \bar{v}=\overline{\sum_{i \in I}} \alpha_{i} \bar{x}_{i}
$$

is invertible in $K\langle H\rangle$. Furthermore, since the homomorphism $G \rightarrow H$ is a homomorphism of ordered groups, we conclude that the minimal element in $\operatorname{Supp} \bar{v}$ is 1 and Lemma 3.1 implies that the same is true for the element $\bar{v}^{-1}$. We can find, therefore, an element $v_{1} \in S$ such that

$$
v v_{1}=1-u, \quad u \in \omega(K N) S .
$$

To prove that the element $v$ is invertible it is enough, via (3.2), to show that $(1-u)^{-1} \in S$. We apply statements (i) and (ii) which were formulated before Lemma 3.1 and conclude that in the infinite series

$$
1+u+u^{2}+\cdots
$$

the elements from a given coset $x N(x \in X)$ can occur in support of a finite number of terms only; this implies first of all that the series (3.3) defines an element of $K\langle G\rangle$, which belongs in fact to $S$. (It is easy to verify that it belongs to $1+\omega(K N) S$.) Finally, the verification of the equation $(1-u)^{-1}=1+u+u^{2}+\cdots$ is routine. 
COROllary 1. Let $\psi: G \rightarrow H$ be a surjective homomorphism of ordered groups. Then there exists a specialization $T: K\langle G\rangle \rightarrow K\langle H\rangle$, extending the homomorphism $K G \rightarrow K H$ of group rings, defined by the homomorphism $\psi$.

Proof. The domain of $T$ is the subring $S$ defined in Proposition 3.1.

COROLlaRY 2. Let $\psi: G \rightarrow H$ be a surjective homomorphism of ordered groups, and let $D$ and $\Delta$ be the subfields of $K\langle G\rangle$ and $K\langle H\rangle$ respectively, generated by $K G$ and $K H$. Then there exists a specialization $\Theta: D \rightarrow \Delta$, extending the homomorphism $\psi$.

Proof. The domain of $\Theta$ is the subring $S \cap D$; and its radical is $J(S) \cap D$.

4.

4.1. Throughout this section let $G$ be a group which contains a series of normal subgroups

$$
G=N_{1} \supseteq N_{2} \supseteq \cdots
$$

such that

$$
\bigcap_{i=1}^{\infty} N_{i}=1 .
$$

Let $F$ be a subgroup of $G$.

LEMMA 4.1. Assume that all the quotient groups $G / N_{i}$ can be ordered in a coherent way, i.e. the homomorphisms $G / N_{i+1} \rightarrow G / N_{i}$ are ordered group homomorphisms. Then the group $G$ can be ordered in such a way that $F$ is an ordered subgroup of it and all the homomorphisms $G \rightarrow G / N_{i}$ and $F \rightarrow F /\left(F \cap N_{i}\right)$ are homomorphisms of ordered groups.

Proof. We consider the subgroup $F / F \cap N_{i}$ as an ordered subgroup of $G / N_{i}$. Let $g$ be an arbitrary element of $G$ and let $i$ be the first index such that the image $\psi_{i}(g)$ in $G / N_{i}$ is nontrivial. If $\psi_{i}(g)>1$ in $G / N_{i}$ we define $g>1$. It is easy to verify that the set $P$ of such elements $g$ is multiplicatively closed and that it is closed with respect to conjugacy in $G$ and that $G=P \cup P^{-1} \cup 1$. Thus we see that $P$ can be taken as a cone of positive elements in $G$ (see [12]) and the assertion follows easily.

COROLlaRY. Let $G$ be an arbitrary group which contains a series of normal subgroups which satisfies conditions (4.1) and (4.2) and let all the groups $G / N_{i}$ be torsion free nilpotent. Then the conclusions of Lemma 4.1 are valid for $G$.

Proof. Lemma 4.1 implies that it is enough to verify that all the quotient groups $G / N_{i}$ can be ordered coherently. But every such quotient group is torsion free nilpotent. It is well known that if $H$ is a torsion free nilpotent group, then we can order it in the following way. Consider a series

$$
H=H_{1} \supseteq \cdots \supseteq H_{k} \supseteq H_{k+1}=1
$$

of isolators of the lower central series. Thus, an element $h \in H_{r}$ if some power of it is in the $r$ th term of the lower central series $(r=1,2, \ldots, k)$. The factors $H_{r} / H_{r+1}$ $(r=1,2, \ldots, k)$ are torsion free abelian groups; we order them in an arbitrary way 
and define that an element $h \in H$ is positive if its first nontrivial factor in the series (4.3) is positive. It is easy to see that we obtain an order on $H$ and that acting in such a way we can order all the quotient groups $G / N_{i}$ coherently. The proof is completed.

4.2. Assume that the group $G$ is ordered in such a way that the homomorphisms $G \rightarrow G_{i}=G / N_{i}$ are homomorphisms of ordered groups. Let $D$ and $D_{i}$ be the subfields of $K\langle G\rangle$ and $K\left\langle G_{i}\right\rangle$, generated by the group rings $K G$ and $K G_{i}$ respectively $(i=1,2, \ldots)$. The results of $\S 3$ imply that every homomorphism $\psi: G \rightarrow G_{i}$ is extended to a specialization $T_{i}: K\langle G\rangle \rightarrow K\left\langle G_{i}\right\rangle$. Furthermore, for every given $i$ we have a homomorphism $\psi_{i}: G_{i+1} \rightarrow G_{i}$ whose kernel is the normal subgroup $N_{i} / N_{i+1}$ of $G_{i+1}=G / N_{i+1}$.

Proposition 4.1. The homomorphism $\psi_{i}$ can be extended to a specialization $K\left\langle G_{i+1}\right\rangle \rightarrow K\left\langle G_{i}\right\rangle$.

Proof. The homomorphisms $G \rightarrow G_{i}$ and $G \rightarrow G_{i+1}$ are homomorphisms of ordered groups. This implies that the homomorphism $\psi_{i}: G_{i+1} \rightarrow G_{i}$ is a homomorphism of ordered groups also. The assertion follows now from Corollary 1 of Lemma 3.1 .

Corollary 1. Every homomorphism $\psi_{i}$ is extended to a specialization $\tau_{i}: D_{i+1} \rightarrow D_{i}$ of $K G$-fields.

Proof. See the proof of Corollary 2 of Proposition 3.1.

Corollary 2. Let $\mathscr{P}_{i}$ be the prime matrix ideal over $K G$ related to the $K G$-field $D_{i}$. Then $\mathscr{P}_{i} \supseteq \mathscr{P}_{i+1}(i=1,2, \ldots)$.

We now consider once again the system of homomorphisms $G \rightarrow G_{i}(i=1,2, \ldots)$. Corollary 1 of Proposition 3.1 implies that every homomorphism $\psi_{i}$ can be extended to a specialization $\Theta_{i}: K\langle G\rangle \rightarrow K\left\langle G_{i}\right\rangle$. The domain $S_{i}$ of $\Theta_{i}$ is the set of elements $s \in K\langle G\rangle$ which have a form

$$
s=\sum_{\alpha} \lambda_{\alpha} x_{\alpha}
$$

where $\lambda_{\alpha} \in K N_{i}$ and $x_{\alpha}(\alpha \in \alpha)$ is a well-ordered subset of a transversal of $N_{i}$ in $G$.

Proposition 4.2. For arbitrary natural $i, S_{i} \subseteq S_{i+1}$.

Proof. Let $s \in S_{i}$. Since $N_{i} \subseteq N_{i+1}$ and the homomorphisms $G \rightarrow G / N_{i}$ are homomorphisms of ordered groups, we can pick a transversal $y_{\beta}(\beta \in \boldsymbol{\beta})$ of $N_{i+1}$ in $N_{i}$ to obtain a transversal $y_{\beta} x_{\alpha}$ of $N_{i+1}$ in $G$. Since $x_{\alpha}(\alpha \in \boldsymbol{\alpha})$ and $y_{\beta}(\beta \in \boldsymbol{\beta})$ are ordered subsets of $G$, so is the set $\left\{y_{\beta} x_{\alpha}\right\}$.

Now take an arbitrary $\lambda_{\alpha}$ in representation (4.4) of $s$ and represent it in the form

$$
\lambda_{\alpha}=\sum_{\beta} \mu_{\alpha \beta} y_{\beta} \quad\left(\alpha \in \alpha, \mu_{\alpha \beta} \in K N_{i+1}\right),
$$


where the number of nonzero coefficients $\mu_{\alpha \beta}$ in (4.5) is finite. Hence, $s$ has a representation

$$
s=\sum_{\alpha, \beta} \mu_{\alpha \beta} y_{\beta} x_{\alpha} \quad\left(\mu_{\alpha \beta} \in K N_{i+1}\right)
$$

and thus $s \in S_{i+1}$.

REMARK. It is worth remarking that we deal in Proposition 4.2 with local subrings $S_{i}$ which define the specializations $\Theta_{i}$ and are constructed in some special way; it might be that some other local subrings of $D$ would define the same specializations but would not satisfy the conclusions of Proposition 4.2.

Proposition 4.3. Let $S=\bigcup_{i=1}^{\infty} S_{i}$. Then $S$ is a subfield of $K\langle G\rangle$ which contains $D$.

Proof. Clearly, $S$ is a subring and we have to prove only that every nonzero element $s \in S$ is invertible. If $s$ is such an element then it belongs to some subring $S_{i}$ and hence has a representation of the form (4.4). Since $\bigcap_{i=1}^{\infty} N_{i}=1$ we can find $i_{0} \geqslant i$ such that at least one of the coefficients $\lambda_{\alpha}$ in (4.4) does not belong to $\omega\left(K N_{i_{0}}\right)$ (in fact, we can find $i_{0}$ such that $\psi_{\alpha}$ does not belong even to the ideal $\omega\left(K N_{i_{0}}\right) K G$ but we do not need this stronger fact). This implies that $s \in S_{i_{0}} \backslash J\left(S_{i_{0}}\right)$ and hence is invertible in $S_{i_{0}}$ and in $S$. Finally, $S$ contains $K G$ and also the subfield $D$, generated by it.

Corollary. Let $T_{i}=D \cap S_{i}(i=1,2, \ldots)$. Then $T_{i}(i=1,2, \ldots)$ is a system of local $K G$-subrings of $D$ which satisfies conditions (2.3)-(2.5).

THEOREM 4.1. Let $\mathscr{P}$ and $\mathscr{P}_{i}$ be the prime matrix ideals related to the KG-fields $D$ and $D_{i}$ respectively. Then $\mathscr{P}_{1} \supseteq \mathscr{P}_{2} \supseteq \cdots$ and $\mathscr{P}=\bigcap_{i=1}^{\infty} \mathscr{P}_{i}$.

Proof. Corollary 2 of Proposition 4.1 implies that $\mathscr{P}_{i} \supseteq \mathscr{P}_{i+1}$, and the corollary of Proposition 4.3 together with 4.2 completes the proof.

5.

5.1. Let $R$ be a domain, $R=A_{1} \supseteq A_{2} \supseteq \cdots$ be a system of ideals such that $\bigcap_{i=1}^{\infty} A_{i}=0$, and assume that every quotient ring $R_{i}=R / A_{i}$ has a field of fractions, say $\Delta_{i}$, such that the natural homomorphism $R_{i+1} \rightarrow R_{i}$ with the kernel $A_{i+1} / A_{i}$ can be extended to a specialization $\Delta_{i+1} \rightarrow \Delta_{i}$. Clearly, if $Q_{i}$ denotes the prime matrix ideal related to the $R$-field $\Delta_{i}$, then $Q_{i} \supseteq Q_{i+1}$. The condition $\bigcap_{i=1}^{\infty} A_{i}=0$ easily implies that $R$ is embedded isomorphically into the ring $\prod_{i=1}^{\infty} R_{i} \subseteq \prod_{i=1}^{\infty} \Delta_{i}$. Let $\mathscr{F}$ be an arbitrary nonprincipal ultrafilter on the set of indices $I=\{1,2, \ldots\}$ and let $\left(\Pi_{i=1}^{\infty} \Delta_{i}\right) / \mathscr{F}$ denote the appropriate ultraproduct. The following fact is well known and we omit its proof.

LEMmA 5.1. The ultraproduct $\left(\prod_{i=1}^{\infty} \Delta_{i}\right) / \mathscr{F}$ is a field and the embedding $R \subseteq \prod_{i=1}^{\infty} \Delta_{i}$ induces an isomorphic embedding of $R$ into $\left(\prod_{i=1}^{\infty} \Delta_{i}\right) / \mathscr{F}$.

THEOREM 5.1. Let $\Delta$ be the subfield of $\left(\prod_{i=1}^{\infty} \Delta_{i}\right) / \mathscr{F}$ generated by the subring $R$, and let $Q$ be the prime matrix ideal related to the $R$-field $\Delta$. Then $Q=\cap_{i=1}^{\infty} Q_{i}$.

COROLlary. Let the conditions of Theorem 5.1 hold. Then the field $\Delta$ is defined up to isomorphism by the system of fields $\Delta_{i}$ and does not depend on the ultrafilter $\mathscr{F}$. 
The proof of Theorem 5.1 is given in the end of the section. For arbitrary natural $i$ we consider the field $\Delta$ and the domain $S_{i+1}$ of the specialization $\Theta_{i+1}: \Delta_{i+1} \rightarrow \Delta_{i}$. Then define $S_{i+2}$ as the inverse image of $S_{i+1}$ under the specialization $\Theta_{i+2}$ : $\Delta_{i+2} \rightarrow \Delta_{i+1}$, etc. Clearly, every subring $S_{i+k}(k=1,2, \ldots)$ is a local subring, containing the radical, of the domain of the specialization $\Theta_{i+k}(k=1,2, \ldots)$. We thus have the inverse system

$$
\Delta \stackrel{\Theta_{i+1}}{\leftarrow} S_{i+1} \stackrel{\Theta_{i+2}}{\leftarrow} S_{i+2} \stackrel{\Theta_{i+3}}{\leftarrow} S_{i+3} \leftarrow \cdots .
$$

Let $S^{(i)}$ be the inverse limit of the system (5.1). Thus, every $S^{(i)}$ is a subring of $\prod_{i=1}^{\infty} \Delta_{i}$ and it is easy to see that $S^{(i)} \subseteq S^{(i+1)}(i=1,2, \ldots)$. We denote by $S$ the subring $\cup_{i=1}^{\infty} S^{(i)}$; it is easy to verify that $R \subseteq S$.

Now let $\bar{X}$ be the image of a subset $X \subseteq \prod_{i=1}^{\infty} \Delta_{i}$ under the homomorphism $\prod_{i=1}^{\infty} \Delta_{i} \rightarrow\left(\prod_{i=1}^{\infty} \Delta_{i}\right) / \mathscr{F}$.

Proposition 5.1. The subring $\bar{S}^{(i)}(i=1,2, \ldots)$ is a local subring of $\left(\prod_{i=1}^{\infty} \Delta_{i}\right) / \mathscr{F}$; its quotient ring by the radical is isomorphic to $\Delta_{i}$.

Proof. Consider the subset of elements $U^{(i)} \subseteq S^{(i)}$ which consists of all the elements whose $i$ th coordinate is zero. Clearly, $U^{(i)}$ is an ideal of $S^{(i)}$. If now an element $\bar{s} \in \bar{S}^{(i)}$ does not belong to $\bar{U}^{(i)}$ we can pick an element $s \in S^{(i)}$ whose image in $\left(\prod_{i=1}^{\infty} \Delta_{i}\right) / \mathscr{F}$ is $\bar{s}$ and whose $i$ th coordinate is nonzero. This implies immediately that the $(i+k)$ th coordinate of $s$ is invertible in $S_{i+k}$ for every $k=1,2, \ldots$ It is well known that an arbitrary ultrafilter contains the complements of finite sets; this implies that the image of $s$ in $\left(\prod_{i=1}^{\infty} \Delta_{i}\right) / \mathscr{F}$ will remain $\bar{s}$ if we replace its first $(i-1)$ coordinates by 1 . We can therefore assume that $s$ is invertible in $\Pi_{i=1}^{\infty} \Delta_{i}$, its inverse is the element $\left(1,1, \ldots, 1, s_{i}, s_{i+1}, \ldots\right)$ and hence $\bar{s}$ is invertible in $\bar{S}^{i} \subseteq\left(\prod_{i=1}^{\infty} \Delta_{i}\right) / \mathscr{F}$. We proved thus that every element $\bar{s} \in \bar{S}^{(i)} \backslash \bar{U}^{(i)}$ is invertible, i.e., $\bar{S}^{(i)}$ is local.

The definition of $U^{(i)}$ implies $S^{(i)} / U^{(i)}$ is isomorphic to $\Delta_{i}$; in order to conclude that $\bar{S}^{(i)} / \bar{U}^{(i)} \simeq \Delta_{i}$ it is enough to observe that $1 \notin \bar{U}^{(i)}$.

We are interested now in the subring $\bar{S}$ of $\left(\prod_{i=1} \Delta_{i}\right) / \mathscr{F}$. The definition of $S^{(i)}$ and $S$ easily imply the relations

$$
\bar{S}^{(i)} \subseteq \bar{S}^{(i+1)} \quad(i=1,2, \ldots) ; \quad \bar{S}=\bigcup_{i=1}^{\infty} \bar{S}^{(i)} .
$$

We conclude from Lemma 5.1 and from the inclusion $R \subseteq S$ that $R \subseteq \bar{S}$.

Proposition 5.2. $\bar{S}$ is a subfield of $\left(\Pi^{\infty} \Delta_{i}\right) / \mathscr{F}$.

Proof. Let $\bar{o} \neq \bar{s} \in \bar{S}$ and let $s \in S$ be an arbitrary element whose image in $\left(\prod_{i=1}^{\infty} \Delta_{i}\right) / \mathscr{F}$ is $\bar{s}$. We can find $i$ such that $s \in S^{(i)}$ and then find $k \geqslant 0$ such that the $(i+k)$ th coordinate of $s$ is nonzero. This implies that $\bar{s}$ is invertible in the local subring $\bar{S}^{i+k}$ of $\bar{S}$.

Corollary. Let $\Delta$ be the subfield of $\left(\prod_{i=1}^{\infty} \Delta_{i}\right) / \mathscr{F}$ generated by $K G$. Then

$$
\Delta=\bigcup_{i=1}^{\infty} \bar{S}^{(i)} \cap \Delta,
$$

where every $\bar{S}^{i} \cap \Delta(i=1,2, \ldots)$ is a local subring of $\Delta$. 
Proof of Theorem 5.1. The proof follows from the corollary of Proposition 5.2 together with Proposition 2.2.

6.

6.1. As in $\S 4$ once again let

$$
G=N_{1} \supseteq N_{2} \supseteq \cdots
$$

be a series of normal subgroups in $G$ such that all the quotient groups $G_{i}=G / N_{i}$ $(i=1,2, \ldots)$ are ordered and $\bigcap_{i=1}^{\infty} N_{i}=1$. We assume (see Lemma 4.1) that $G$ is ordered in such a way that the homomorphisms $G \rightarrow G_{i}$ are homomorphisms of ordered groups and once again denote by $D$ and $D_{i}$ the subfields of $K\langle G\rangle$ and $K\left\langle G_{i}\right\rangle$ generated by $K G$ and $K G_{i}$ respectively. We recall that the $K G$-field $D_{i}$ is defined by the prime matrix ideal $\mathscr{P}_{i}(i=1,2, \ldots)$. Let $\mathscr{F}$ be an arbitrary ultrafilter on the set of indices $\{1,2, \ldots\}$ and let $\Delta$ be the subfield of $\left(\prod_{i=1}^{\infty} D_{i}\right) / \mathscr{F}$, generated by subring $K G$ (see Lemma 5.1).

THEOREM 6.1. The $K G$-fields $D$ and $\Delta$ are isomorphic.

Proof. Theorem 5.1 implies that $\Delta$ is defined by the prime matrix ideal $\cap_{i=1}^{\infty} \mathscr{P}_{i}$. Theorem 4.1 implies that the same is true for the field $D$. Hence $D$ and $\Delta$ are isomorphic.

6.2. We assume throughout this section that the group $G$ is residually torsion free nilpotent and that the series (6.1) is such that all the quotient groups $G / N_{i}$ $(i=1,2, \ldots)$ are torsion free nilpotent (and $\cap_{i=1}^{\infty} N_{i}=1$ ). Corollary of Lemma 4.1 implies that we can assume that $G$ is ordered in such a way that the homomorphisms $G \rightarrow G_{i}(i=1,2, \ldots)$ are homomorphisms of ordered groups. It is worth remarking that the field $D_{i}$ in this case is the field of Ore fractions of the group ring $K G_{i}$ of a torsion free nilpotent group $G_{i}$.

Now let $H_{j}(j=1,2, \ldots)$ be the second series in $G$ with the same properties as the series $N_{i}$. We obtain a second order in $G$, such that $G \rightarrow G / H_{j}(j=1,2, \ldots)$ are homomorphisms of ordered groups and a subfield $D^{\prime}$, generated by $K G$ in the new Malcev-Neumann power series ring. Let $D_{j}^{\prime}$ denote the field of fractions of the group ring $K\left(G / H_{j}\right)$.

THEOREM 6.2. The R-fields $D$ and $D^{\prime}$ are isomorphic.

We first need the following fact.

LEMMA 6.1. Let $G$ be a group, and $N \supseteq H$ be two normal subgroups of $G$ such that $G / N$ and $G / H$ are torsion free nilpotent groups. Let $\Delta_{1}$ and $\Delta_{2}$ be the Ore fields of fractions of the group rings $K(G / N)$ and $K(G / H)$ respectively and $A_{i}$ be the prime matrix ideals, related to the $K G$-fields $\Delta_{i}(i=1,2)$. Then $A_{1} \supseteq A_{2}$.

Proof. We have a homomorphism $\psi: G / H \rightarrow G / N$ with the kernel $N / H$ and we also denote by $\psi$ the corresponding homomorphism of group rings $K(G / H) \rightarrow$ $K(G / N)$. The torsion free nilpotent groups $G / H$ and $G / N$ can be ordered in such a way that $\psi$ is a homomorphism of ordered groups and Corollary 2 of Proposition 3.1 implies now that $\psi$ can be extended to a specialization from $\Delta_{2} \rightarrow \Delta_{1}$. We obtain from this that $A_{1} \supseteq A_{2}$. 
Proof of Theorem 6.2. Let $\mathscr{P}_{i}$ and $Q_{j}$ be the prime matrix ideal related to the field $D_{i}$ and $D_{j}^{\prime}$ respectively. Theorem 4.1 implies that

$$
\mathscr{P}_{1} \supseteq \mathscr{P}_{2} \supseteq \cdots ; \quad Q_{1} \supseteq Q_{2} \supseteq \cdots
$$

and we have to prove that $\bigcap_{i=1}^{\infty} \mathscr{P}_{i}=\bigcap_{j=1}^{\infty} Q_{j}$. A routine argument reduces the proof to the case when the group $G$ is finitely generated. It is easy to verify that in this case for every given $j$ we can find an index $i=i(j)$ such that $N_{i(j)} \subseteq H_{j}$ and thus $\mathscr{P}_{i(j)} \supseteq Q_{j}$. Hence

$$
\bigcap_{i=1}^{\infty} \mathscr{P}_{i} \supseteq \bigcap_{j=1}^{\infty} Q_{j}
$$

The inverse inclusion is also true; hence

$$
\bigcap_{i=1}^{\infty} \mathscr{P}_{i}=\bigcap_{j=1}^{\infty} Q_{j}
$$

and the proof is completed.

Corollary 1. Let $\left(\prod_{i=1}^{\infty} D_{i}\right) / \mathscr{F}$ and $\left(\prod_{j=1}^{\infty} D_{j}^{\prime}\right) / \mathscr{F}^{\prime}$ be two ultraproducts. Then the subfields, generated by the subring $K G$ in each of them, are isomorphic.

Proof. The proof follows from Theorems 6.1 and 6.2.

Now let $G_{1}$ and $G_{2}$ be two residually torsion free nilpotent groups, $N_{i}(i=1,2, \ldots)$ and $H_{j}(j=1,2, \ldots)$ be descending series in $G_{1}$ and $G_{2}$ respectively such that

$$
\bigcap_{i=1}^{\infty} N_{i}=1 ; \quad \bigcap_{j=1}^{\infty} H_{j}=1,
$$

and all the quotient groups $G_{1} / N_{i}$ and $G_{2} / H_{j}$ are torsion free nilpotent. We assume too that $G_{1}$ and $G_{2}$ are ordered in such a way that all the homomorphisms $G_{1} \rightarrow G_{1} / N_{i}$ and $G_{2} \rightarrow G_{2} / H_{j}$ are homomorphisms of ordered groups and let $K\left\langle G_{1}\right\rangle$ and $K\left\langle G_{2}\right\rangle$ be the corresponding Malcev-Neumann power series rings. We consider two subgroups $F_{\alpha} \subseteq G_{\alpha}(\alpha=1,2)$ and let $\psi: F_{1} \rightarrow F_{2}$ be an isomorphism between them. We have the following easy corollary of Theorem 6.2.

COROLlaRY 2. Let $\Delta_{\alpha}$ be the subfield of $K\left\langle G_{\alpha}\right\rangle$ generated by the group ring $K F_{\alpha}$ $(\alpha=1,2)$. Then the isomorphism $\psi$ is extended to an isomorphism between $\Delta_{1}$ and $\Delta_{2}$.

PROOF. The corollary of Lemma 4.1 implies first of all that $F_{\alpha}$ is an ordered subgroup of $G_{\alpha}(\alpha=1,2)$ and we obtain therefore a natural imbedding $K\left\langle F_{\alpha}\right\rangle \subseteq$ $K\left\langle G_{\alpha}\right\rangle(\alpha=1,2)$. We now consider the groups $F_{1}$ and $F_{2}$ as two isomorphic copies of a group $F$ and the fields $K\left\langle F_{1}\right\rangle$ and $K\left\langle F_{2}\right\rangle$ as the Malcev-Neumann fields for $K F$ defined by two different series in $F$, which are obtained from $N_{i} \cap F$ ( $i=$ $1,2, \ldots)$ and $H_{j} \cap F(j=1,2, \ldots)$. Theorem 6.2 now implies that $K F$ generates isomorphic subfields $\Delta_{1}$ and $\Delta_{2}$ in $K\left\langle F_{1}\right\rangle$ and $K\left\langle F_{2}\right\rangle$ respectively.

We can now prove Theorem 6.3. 
THEOREM 6.3. Let $G_{1}$ and $G_{2}$ be two residually torsion free nilpotent groups, and let $G=G_{1}{ }_{F} G_{2}$ be their amalgamated free product. Then the group ring $K G$ can be embedded in a field.

Proof. We use the same notations as in Corollary 2 of Theorem 6.2. Thus let $\Delta_{\alpha}$ be the subfield of $K\left\langle G_{\alpha}\right\rangle$ generated by the group ring $K F_{\alpha}(\alpha=1,2)$. Since $\Delta_{1}$ and $\Delta_{2}$ are isomorphic we can consider the coproduct $R=K\left\langle G_{1}\right\rangle{ }_{\Delta} K\left\langle G_{2}\right\rangle$, amalgamating the subfields $\Delta_{\alpha} \subseteq K\left\langle G_{\alpha}\right\rangle(\alpha=1,2)$ and our argument from this moment is the same as Lewin's one (see [1]). By Cohn's theorems [2 and 3] $R$ is a fir and can be embedded into a field. It is important that different coset representations of $G_{\alpha}$ and $F_{\alpha}$ are left linearly independent elements of $K\left\langle G_{\alpha}\right\rangle$ over $K\left\langle F_{\alpha}\right\rangle$ and hence over $\Delta_{\alpha}$ $(\alpha=1,2)$; we can therefore obtain a left basis of $K\left\langle G_{\alpha}\right\rangle$ over $\Delta$ which contains a transversal for $F_{\alpha}$ in $G_{\alpha}(\alpha=1,2)$. But it is known (see [2]) that the monomials in these basis elements with successive terms from different factors form a left basis for $R$ over $\Delta$. This implies easily that the group ring $K G$ of the group $G=G_{1} *_{F} G_{2}$ is embedded isomorphically in $R$ and hence in a field.

COROllary. Let $\mathrm{H}$ be a residually torsion free nilpotent group, $\mathrm{H}_{1}$ and $\mathrm{H}_{2}$ be two isomorphic subgroups of it, and $G=\left\langle H, t, t^{-1} H_{1} t=H_{2}\right\rangle$ be the appropriate $H N N$ extension of $H$. Then the group ring $K G$ is a domain and can be embedded in a field.

Proof. Let $Q=H * U$ and $L=H * V$, where $U$ and $V$ are infinite cyclic groups. The groups $Q$ and $L$ are residually torsion free nilpotent since they are free products of residually torsion free nilpotent groups (see [13]). But the $\mathrm{HNN}$-extension, $G$ is embedded into an appropriate amalgamated free product of $Q$ and $L$ (see [14, vol. 2, p. 53]) and the assertion now follows from Theorem 6.3.

Acknowledgment. We would like to thank the referee and Professor P. M. Cohn whose suggestions and criticism helped to improve the exposition of the article and to correct some inaccuracies and errors.

\section{REFERENCES}

1. J. Lewin, Fields of fractions for group algebras of free groups, Trans. Amer. Math. Soc. 190 (1974), 339-346.

2. P. M. Cohn, On the free products of associative rings. III, J. Algebra 8 (1968), 376-383.

3. __ The embeddings of firs in skew fields, Proc. London Math. Soc. (3) 23 (1971), 193-213.

4. J. Lewin and T. Lewin, An embedding of the group algebra of a torsion free one relator group in a field, J. Algebra 52 (1978), 39-79.

5. P. M. Cohn, Free rings and their relations, Academic Press, New York, 1971.

6. P. Malcolmson, A prime matrix ideal yields a skew field, J. London Math. Soc. 18 (1978), 221-233.

7. A. I. Lichtman, On normal subgroups of multiplicative groups of skew fields generated by a polycyclic-by-finite group, J. Algebra 78 (1982), 548-577.

8. On linear groups over a field of fractions of a polycyclic group ring, Israel Math. J. 42 (1982), $318-326$.

9. On matrix rings and linear groups over a field of fractions of enveloping algebras and group rings. I, J. Algebra 88 (1984), 1-37.

10. On matrix rings and linear groups over a field of fractions of enveloping algebras and group rings. II, J. Algebra 90 (1984), 516-526. 
11. Free subgroups in linear groups over some skew fields, J. Algebra (to appear).

12. D. S. Passman, The algebraic structure of group rings, Wiley-Interscience, New York, 1977.

13. A. I. Malcev, Generalized nilpotent algebras and their adjoint groups, Mat. Sb. 25 (67) (1949), 347-366: English transl., Amer. Math. Soc. Transl. 69 (1968), 7-23.

14. A. G. Kurosh, The theory of groups, Chelsea, New York, 1956.

Department of Mathematics, The Hebrew University, Jerusalem, Israel

Departmfent of Mathematics, University of Wisconsin at Parkside, Kenosha, Wisconsin 53140 